\title{
Rate Dynamics and Transmission Algorithms in Clustered Sensor Networks
}

\author{
${ }^{1}$ A.T. Burrel ${ }^{2}$ P. Papantoni-Kazakos \\ ${ }^{1}$ Dept. of Computer Science, Oklahoma State University, USA \\ ${ }^{2}$ Dept. of Electrical Engineering, University of Colorado Denver, USA \\ tburrell@okstate.edu; titsa.papantoni@ucdenver.edu
}

\begin{abstract}
We consider wireless clustered sensor networks consisting of large numbers of life-limited sensors, where the size of each cluster is determined by the data rate generated by the sensors it contains. The limited sensors' life-span and their possible mobility induce variations in the sensor population and data rate dynamics. The variations of the sensors' population necessitate the deployment of random access data transmission algorithms, where stable such algorithms lie within the limited sensing class. We propose the deployment of the Limited Sensing Stack Random Access Algorithms (LSSRAAs), with a modification which allows the accommodation of high priority data. We evaluate the performance of the LSSRAAs in the presence of sensor expirations and recommend design specifications for systems with low versus relatively high energy reserves.
\end{abstract}

Keywords: Clustered Sensor Networks; dynamic data rates; limited sensor life span; limited sensing random access transmission algorithms; priority data; dynamic cluster reconfiguration.

\section{Introduction}

We consider wireless sensor networks containing large numbers of possibly mobile sensors, normally distributed over a wide geographical area. Such networks are then comprised of sensor clusters, where the size of each cluster may be determined by the communication range of the devices, in conjunction with the aggregate data rate generated by the sensors contained in the cluster. The clusters are generally connected via a backbone network which also normally includes a base station that performs global data processing operations.

The distinguishing feature in wireless sensor networks is the limited life-spans of the sensors, as induced by their energy consumption. Interesting results focusing on energy consumption have been obtained by several researchers: Bounds on energy conservation techniques have been derived in [1], role assignments targeting energy conservation have been developed in [2], energy conservation routing techniques have been proposed in [3], [4], [5] and issues arising due to energy conservation have been discussed in [5], [6]. In addition, topological and node-cooperation issues have been included in [7], [8], [9], while approaches to performance monitoring have been presented in [10]. An interesting rate allocation algorithm has been presented in [11], which is based on a modification of the max-min routing in [12] and the lexicographic linear programming approach in [13]. In [14], a dynamic rate 
A.T. Burrell \& P. Papantoni-Kazakos; Rate Dynamics and Transmission Algorithms in Clustered Sensor Networks, Transactions on Networks and Communications, Volume 2 No 5, Oct (2014); pp: 45-59

allocation methodology has been presented that is facilitated by the powerful data rate monitoring algorithm in [15], [16],[17], while an architectural reconfiguration approach based on data rate monitoring is presented in [18] and [19]. In [20], energy efficient clustering algorithms are proposed, including a discussion on LEACH approaches, where energy consumption is assumed to be strictly a function of geographical distances and where transmission collisions are completely ignored. Considering transmission protocols, those proposed or partially implemented (in Zigbee, IEEE802.15.4 etc.) within the Random Access class are ALOHA-based and are characterized by well-known instabilities that pull the throughput down rapidly to zero, as the user population increases.

Wireless sensor networks are designed to satisfy signal processing objectives. Thus, their performance metrics are determined by those of the latter objectives [21], [22]. When time constraints are imposed on high accuracy signal processing operations, the consequence is increased required overall data rates. At the same time, due to sensor expirations and subsequent time-varying sensor populations, the data transmitting algorithms deployed by the sensors must be within the random access class. In particular, for stability and implementability, the class of Limited Sensing Random Access Algoriths (LSRAAs) must be then deployed by the sensors in each cluster. Such are the algorithms in [23], [24], [25], [26], whose performance is a function of the their input data rates, while, at the same time, the life-span of the sensors is a function of the data rates generated within the clusters containing them, [27], [3], [4], [11]. Thus, required overall data rates, in conjunction with rate-dependent LSRAA performance and sensor life-spans, necessitate network-architecture and network-operations adaptations, so that the sensors' survivability limitations do not interfere with the required network overall performance, [2], [9].

In this paper, we study the deployment of Limited Sensing Stack Random Access Algorithms (LSSRAAs) in clustered wireless sensor networks containing high priority sensors, where all sensors have a limited lifespan. The present study is an extension of that performed in [19]. We model sensor expiration, deploy LSSRAAs which give a delay advantage to high priority data and analyze system delays and sensor expiration rates. The organization of the paper is as follows: In Section 2, we present the system model. In Section 3, we outline the operations of the deployed LSSRAAs and summarize their performance in the absence of sensor expirations. In Section 4, we present numerical performance evaluations of the multi-cluster system in the presence of sensor expirations. In Section 5, we draw conclusions.

\section{System Model}

The overall system is depicted in Figure 1 and is comprised of multiple clusters connected with each other via a backbone network of Aggregate Forward Nodes (AFNs) and a Base Station (BS). Each cluster contains a large sensor population whose identities vary in time due to either mobility or expiration or both. Such a sensor population necessitates the deployment of a Random Access Algorithm (RAA) by the sensors for their data transmission within the cluster which contains them [23], [27]. The data generated across all clusters are in the form of identical length packets. Each sensor is a limited energy device whose stored energy may be exhausted by the retransmission and feedback sensing operations required for the transmission of a single packet (as dictated by the deployed RAA). In our model, we thus assume that each sensor generates a single packet and expires: if its stored energy is exhausted and the sensor expires before the successful transmission of the generated packet, the latter is lost/rejected; it is successfully transmitted, instead, if successful transmission precedes energy exhaustion and sensor expiration. The large sensor population, in conjunction with the model of a single 
packet generated independently per sensor, gives rise to the Limit Poisson User Model per cluster [23], [27]. That is, the packet traffic per cluster is modeled as being generated by infinitely many independent Bernoulli users whose aggregate generated packet traffic is a Poisson process, where each packet is an independent user.

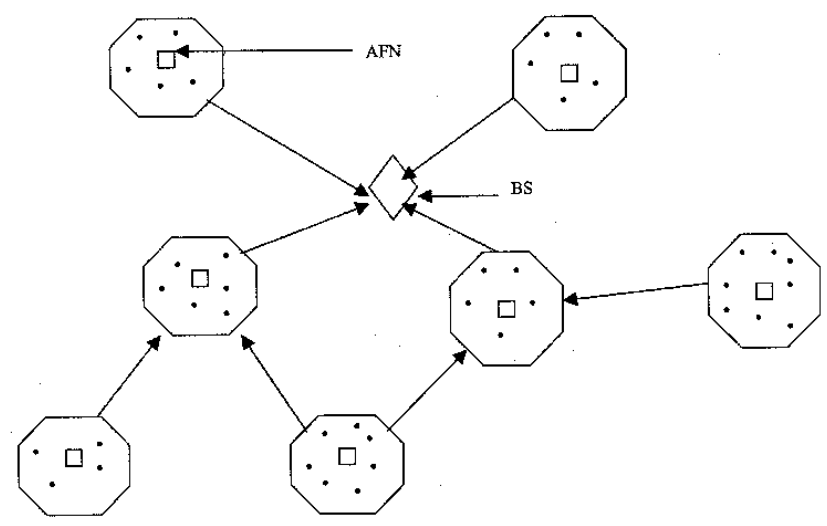

Figure 1: System Topology

It is assumed that each cluster in the network includes a distinct dedicated transmission channel (distinct dedicated frequency band) and contains two classes of sensors: regular and high priority. The regular sensors produce regular data and are not located close to the cluster boundaries. The high priority sensors are either located close to the cluster boundaries and may move shortly to the transmission range of another cluster, or they generate high priority data. The regular sensors monitor and transmit through only the transmission channel of the cluster they are contained in, while the high priority sensors may in addition monitor and transmit through the transmission channels of neighboring clusters. As we will see in Section III, the purpose of this additional channel monitoring employed by the high priority sensors is delay reduction, while their energy consumption may be simultaneously accelerated.

Each cluster transmission channel accommodates synchronous transmissions, where the time of all channels across the various clusters is slotted synchronously to identical length slots, each corresponding to a single packet transmission. Time is measured in slot units, where slot $t$ occupies the time interval $(\mathrm{t}-1, \mathrm{t}]$. Slot feedback outcomes are assumed binary Collision (C) vs. Non-Collision (NC) across all cluster transmission channels: that is, it is assumed that a sensor monitoring a transmission channel may distinguish accurately between collision versus non-collision per channel slot, where collision indicates the simultaneous transmission by at least two packets, while non-collision indicates that a slot is either unoccupied or it is occupied with a single packet transmission. In addition, a collision results in complete loss of all involved packets requiring subsequent retransmissions, while a NC slot occupied with a single packet implies noiseless transmission. Given the transmission channel of a cluster, we will denote by $x_{t}$ the feedback outcome ( $C$ vs. NC) of channel slot $t$.

Sensors transmit via given cluster channels deploying a stable Random Access Algorithm (RAA). As we will discuss in detail in Section III, the operations of such an RAA impose initialization periods during 
A.T. Burrell \& P. Papantoni-Kazakos; Rate Dynamics and Transmission Algorithms in Clustered Sensor Networks, Transactions on Networks and Communications, Volume 2 No 5, Oct (2014); pp: 45-59

which a data generating sensor only monitors passively the feedback outcomes of the channels, until the sensor enters a Collision Resolution Interval (CRI) within a single channel. During this CRI, the sensor both monitors feedback outcomes and occasionally retransmits, until the time when a successful retransmission occurs. As we will discuss in detail in Section III, for regular sensors, the initialization period and the CRI both involve only one channel: the transmission channel of the cluster that contains them. For high priority sensors, on the other hand, $M>1$ channels are involved in the initialization period; the transmission channel of the sensor's home cluster plus the transmission channels of M-1 neighboring clusters, while the CRI still involves a single transmission channel selected by the sensor among the above $\mathrm{M}$ channels via some selection process (explained in Section III).

As in [19], we adopt a linear model for the energy consumption by each sensor in the system, as expressed by inequality (1) below, where we first define:

$\beta$ : The amount of per sensor energy consumed by the monitoring of a single channel slot.

$\eta$ : The amount of per sensor energy consumed by a single packet (re) transmission.

$\tau_{1}$ : The number of slots during which a sensor passively monitors $M$ channels in the system without attempting transmissions ( $M>1$ for high priority sensors).

$\tau_{2}$ : The number of slots during which a sensor monitors a single channel in the system, while participating in a Collision Resolution Interval (CDI).

m: The number of transmissions during the CRI the sensor participates in.

$\xi$ : $\quad$ The total energy stored in a single sensor for packet transmission.

Using the above notation, we assume that the sensor expires, with subsequent loss/rejection of its generated packet, If:

$$
\beta\left(\mathrm{M} \tau_{1}+\tau_{2}\right)+\eta \mathrm{m}>\xi
$$

We note that in the above expression we have assumed that the energies that a high priority sensor consumes for either monitoring or transmitting through a neighboring transmission channel are the same with those corresponding to its local transmission channel. This assumption may be untrue is some cases, where considerable variations in communication range may cause increased energy consumption when neighboring transmission channels are considered. In the latter cases, the quantities $\beta$ and $\eta$ defined above will be channel dependent, instead.

\section{The Deployed LSSRAAs}

As discussed in Section 2, the sensor population per cluster is time varying due to mobility and expirations. Thus, the sensor identities are not known to the system at all times. This gives rise to the unknown user population model which necessitates the deployment of Random Access Algorithms (RAAs) for transmission. At the same time, the per cluster Limit Poisson User Model adopted in Section II excludes the deployment of ALOHA-based RAAs, since the latter induce throughput zero then, [28], [29]. We propose, instead, the deployment of the Limited Sensing Stack Random Access Algorithms (LSSRAAs) in [23], in conjunction with the selection policy in [25] for the high priority sensors. Below, we describe the proposed LSSRAAs and the selection policy, for clusters containing both regular and high 
priority sensors, where, in addition to their local transmission channel, the high priority sensors may also access a number of neighboring cluster transmission channels.

\subsection{LSSRAA Operations for Regular and High Priority Sensors}

Let us consider a total of $\mathrm{M}$ distinct clusters and their corresponding transmission channels, indexed by $\mathrm{j}$ $=1, \ldots, M$. Let us then denote by $x t(j) ; j=1, \ldots, M$ the feedback outcome of slot $t$ in channel $j$. The regular sensors in cluster $\mathrm{j}$, only monitor the feedback outcomes $\mathrm{xt}(\mathrm{j})$ and transmit only through channel $\mathrm{j}$. The high priority sensors in cluster $\mathrm{j}$ monitor, instead, all channel feed backs $x t(j) ; j=1, \ldots, M$ and select dynamically one of the $M$ channels for transmission. The LSSRAAs in [23], in conjunction with the selection policy in [25], induce an initialization and a collision resolution processes, where the initialization process is different for regular versus high priority sensors. We will first explain the collision resolution process, since it actually dictates the initialization process.

\subsection{Collision Resolution Process}

The collision resolution process induces a sequence of subsequent Collision Resolution Intervals (CRIs) per transmission channel whose characteristics are dictated by the operations of the deployed RAA. In the case of the LSSRAAs in [23] and the Limit Poisson User Model, a parameter $\Delta$ is utilized which corresponds to the size of the arrival interval resolved by each CRI (packet arrivals within such arrival interval transmitted successfully during the CRI), where the value of $\Delta$ is optimized for throughput maximization. The placement of the $\Delta$-size window on the arrival access is determined asynchronously by the sensors, as will be explained below in this section, and is named the examined interval of the CRI.

The LSSRAAs in [23] are algorithms whose collision resolution process may be depicted by a stack containing a finite number of cells. Let us consider such an algorithm which may be described by a K-cell stack. Then, during some CRI on a given channel, each participating sensor follows the collision resolution rules by utilizing a counter whose values lie in the set of integers, $[1,2, \ldots \mathrm{K}]$. We denote by $r_{t}$ the counter value of some participating sensor at slot $t$. The $K$ different possible values of the counter place the user in one of the $\mathrm{K}$ cells of a K-cell imaginary stack. When its counter value is 1 , the sensor transmits; it withholds at K-1 different stages otherwise. When a CRI begins, all sensors whose packet arrivals lie in the $\Delta$-length examined interval of the CRI set their counters at 1 ; thus, they all transmit within the first slot of the CRI. If the examined interval contains at most one packet, the first slot of the $\mathrm{CRI}$ is a non-collision slot and the CRI lasts one slot. If the examined interval contains at least two packets, instead, the CRI starts with a collision which is resolved within the duration of the CRI (all packets involved in the initial collision are successfully transmitted during the CRI) via the following rules, where $x_{t}$ denotes the feedback outcome of the channel's slot $t$ :

The sensor transmits in slot $t$, if and only if $r_{t}=1$. The packet of a sensor is successfully transmitted in slot $t$, if and only if $r_{t}=1$ and $x_{t}=N C$.

The counter values transition in time during the CRI, as follows:

$$
\begin{aligned}
& \text { If } x_{t-1}=N C \text { and } r_{t-1}=j ; j=2,3, . ., K \text {, then } r_{t}=j-1 \\
& \text { If } x_{t-1}=C \text { and } r_{t-1}=j ; j=2,3, \ldots K \text {, then } r_{t}=j \\
& \text { If } x_{t-1}=C \text { and } r_{t-1}=1 \text {, then, } r_{t}=
\end{aligned}
$$
$1 ;$ w.p. $1 / \mathrm{K}$
2 ;w.p. $1 / \mathrm{K}$
3 ; w.p. 1/K 


$$
\text { K ; w.p. } 1 / \mathrm{K}
$$

From the above rules, it can be seen that, on a given cluster transmission channel, a CRI which starts with a collision slot ends with $\mathrm{K}$ consecutive non-collision slots, an event which can not occur at any other instant during the CRI. Thus, the observation of $\mathrm{K}$ consecutive non-collision slots signals the certain end of a CRI to all users in the system; it either signifies the end of a CRI that started with a collision or the end of a sequence of $\mathrm{K}$ consecutive length-one CRIs. Therefore, a sensor which arrives in the cluster without any knowledge of the cluster-channel feedback history can synchronize with the local LSSRAA's algorithmic operations upon the observation of the first K-tuple of consecutive noncollision slots. This observation leads to the asynchronous placement of the size- $\Delta \mathrm{CRI}$ examined intervals' on the arrival axis that describes the initialization process, for the regular and the high priority sensors.

\subsection{Initialization Process}

In general, if a CRI on a given cluster transmission channel ends with slot $\mathrm{t}$, the examined interval of the next CRI on the channel is selected with its right most edge placed $\mathrm{K}-1$ slots to the left of slot $\mathrm{t}$ and it contains those packets whose local updates (updates corresponding to the transmission channel of the given cluster) fall in the interval ( $\mathrm{t}-\mathrm{K}+1-\Delta, \mathrm{t}-\mathrm{K}+1)$. The generation of the updates is explained below

For regular sensors in a given cluster, the updates $\left\{\mathrm{t}^{\mathrm{k}}\right\}$ of a packet (one packet corresponds to one sensor) are generated strictly for the local transmission channel (that corresponding to the cluster where the sensors reside) and are as follows: Let $t_{0}$ be the slot within which a packet is generated. Then, define $t^{0}$ to be equal to $t_{0}$. Starting with slot $t^{0}$, the corresponding regular sensor senses continuously the feedback outcomes of the local transmission channel. It does so passively, until it observes the first $K$-tuple of consecutive $N C$ slots, ending with slot $t_{1}$. If $t^{0} \in\left(t_{1}-K+1-\Delta, t_{1}-K+1\right)$, the sensor participates in the CRI which starts with slot $t_{1}+1$ on the local channel. Otherwise, it updates its arrival instant to $\mathrm{t}^{1}=\mathrm{t}^{0}+\Delta$ and observes local feedback outcomes passively until the end of the latter $C R I$, ending with slot $t_{2}$. If $t^{1} \in\left(t_{2}-K+1-\Delta, t_{2}-K+1\right)$, the sensor participates in the CRI which starts with slot $t_{2}$; otherwise, the sensor updates its arrival instant by $\Delta$ again and repeats the above process. In general, if $\left\{t_{n}\right\} n \geq 1$ denotes the sequence of consecutive local CRI endings since the first K-tuple of consecutive NC slots, the sensor participates in the $k^{\text {th }} C R I$ if $t^{k-1} \in\left(t_{k}-K+1-\Delta, t_{k}-K+1\right)$ and $t^{n} \notin\left(t_{n+1}-K+1\right.$ $\left.\Delta, t_{n}-K+1\right)$; for all $n \leq k-2$. In the latter case, $t_{k}$ denotes the beginning slot of the first after the packet's arrival CRI whose examined interval the packet's update falls into.

For high priority sensors in any of the $\mathrm{M}$ clusters, the initialization process develops as follows. Upon generating a packet, each high priority sensor starts observing continuously the feedback outcomes from all $\mathrm{M}$ transmission channels in the $\mathrm{M}$ clusters. Subsequently, the sensor generates $\mathrm{M}$ sequences of updates, separately for each channel, as described in the above paragraph, imagining itself as a regular user in each cluster. The high priority sensor participates then in the first across all M channels CRI whose examined interval its corresponding update falls into and transmits its packet successfully during the process of the latter. This dynamic CRI participation constitutes the high priority sensor's selection policy. When $\mathrm{m}$ updates (across $\mathrm{m}$ channels) of a high priority sensor fall within the examined intervals of $m$ simultaneously starting CRIs (across $m$ channels), one of the $m$ CRIs is selected equiprobably. 


\subsection{Performance Characteristics in the Absence of Sensor Expirations}

In the absence of sensor expirations and of high priority sensors in M clusters, the LSSRAAs in this section give rise to $M$ identical and independent RAA systems. As found in [23], the throughput of each such system, for the Limit Poisson User Model, is then 0.43 (in average number of packets per slot) for all $\mathrm{K}$ values in the stack, where throughput is defined as the highest traffic Poisson rate for which the system is stable. For different $\mathrm{K}$ values, the 0.43 throughput is attained, however, for different values of the window size $\Delta$. In [23], it was found that for $\mathrm{K}=2$ the optimal window size is $\Delta^{*}=2.33$, while for $\mathrm{K}=3$ the optimal window size is $\Delta^{*}=2.56$.

In the absence of sensor expirations and the presence of high priority sensors in M participating clusters, their dynamic selection policy causes coupling of the RAA operations across the M cluster transmission channels. The result is throughput reduction of the $M$ channel system, at the gain of reduced delays for the high priority traffic. An outline of the throughput evaluation process for any $K$ value LSSRAA, in the presence of the Limit Poisson User Model can be found in [19]. In Figure 2, we plot, as an example, the stability regions induced for the LSSRAA with $\mathrm{K}=3$ and $\mathrm{M}=2$ clusters, as found in [25]. The latter figure corresponds to Limit Poisson Models for the two regular traffics (local to clusters 1 and 2 with corresponding Poisson rates $\lambda_{1}$ and $\lambda_{2}$ ), as well as for the high priority traffic (whose Poisson rate is $\lambda_{3}$ ). We note that, for $M=2$ and changing $K$ values, the stability regions in the figure will remain unchanged, as long as the corresponding to the chosen $\mathrm{K}$ value optimal window size $\Delta$ is used. Regarding delays, they change when either one of the $M$ or $K$ values varies. For $K$ fixed, as $M$ increases, the per channel throughput decreases, at the gain of enhanced delay reductions for the high priority traffic [25]. Quantitative delay results will be presented in Section 4.

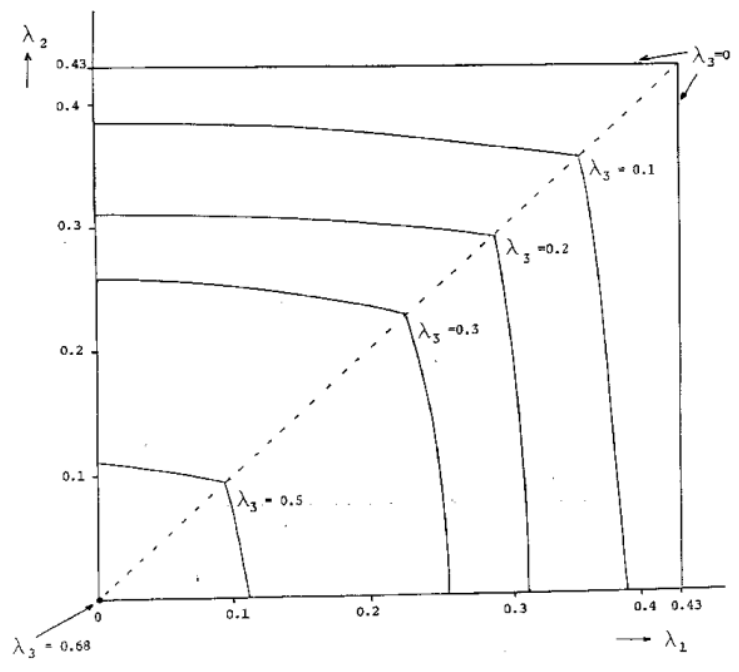

Figure 2: Stability Regions for $\mathrm{K}=2$ and $\mathrm{M}=\mathbf{2}$

\subsection{Performance and Dynamics in the Presence of Sensor Expirations}

In the presence of sensor expirations, sensors may expire before the successful transmission of their generated packet, causing packet losses/rejections. System stability becomes then a void concept; thus, so does throughput. The relevant system performance criteria for both the regular and the high priority traffics are then: rejection rates (percentage of rejected traffic) and delays of the successfully 
A.T. Burrell \& P. Papantoni-Kazakos; Rate Dynamics and Transmission Algorithms in Clustered Sensor Networks, Transactions on Networks and Communications, Volume 2 No 5, Oct (2014); pp: 45-59

transmitted packets, all as functions of the input Limit Poisson traffic rates. In Section IV below, we include simulated such performance results, for Limit Poisson input traffics with varying rates and the sensor expiration model in (1) with various parameter values.

The deployed LSSRAAs, in conjunction with the power consumption formula for the sensors, induce specific packet rejection rates and specific delays of the successfully transmitted packets, as functions of the input traffic rates. As a result, required constraints on packet rejection rates and delays dictate then the acceptable operational rate regions of the input traffics. Subsequently, the latter regions determine the size of each cluster, where cluster size refers to the aggregate data rate generated in it rather than its geographical area. We will discuss specific such arising cluster size issues in Section 4.

\section{Numerical Performance Evaluations}

We simulated the case of seven clusters indexed from 1 to 7 . We assumed that each cluster contains the same fraction $\alpha$ of high priority users and that each such user monitors the same number M-1 of neighboring transmission channels (in addition to that in its own cluster). Visualizing the placement of clusters 1 to 7 on a circle, a high priority user monitors $n$ neighboring channels in each one of the clockwise and counterclockwise directions; if $M-1=2 n$, while it monitors $n+1$ in the clockwise and $n$ in the counterclockwise directions neighboring channels; if $M-1=2 n+1$. All traffics have been modeled as Poisson, where system performance in both the presence and the absence of sensor expirations has been evaluated. In the presence of sensor expirations, various values of the parameters $\beta, \eta$ and $\xi$ in the expiration formula (1) have been considered, while, here, we present the results for a representative selection subset. For both the regular and the high priority traffics, we present expected delay results in all cases, while we also present rejection rates results, when the sensor expiration formula is active.

In both the presence and the absence of sensor expirations, we tested the following high priority traffic fraction values: $\alpha=0.05,0.1,0.3,0.5$. We also then tested the following values for the number of channels monitored by high priority users: $M=2, M=3, M=4, M=5$. In the presence of sensor expirations, the selected sets $[\beta, \eta, \xi]$ of constants' values in formula (1) were: $[5,30,300],[5,10,100],[5,50,500]$ and $[5,50,1000]$.

In the absence of sensor expirations, representative results are depicted by Figures 3 and 4 . In the figures' legend, 7-k-R/S-L/M-NE, 7 depicts the 7 channel system, $k=M-1, R$ stands for regular while $S$ stands for high priority users, L/M depicts the fraction of high priority traffic (0.05 / 0.3) and NE stands for absence of energy constraints. In Figure 3, expected delays for both the regular and the high priority users are plotted against cluster traffic rates, when the fraction $\alpha$ of high priority traffic per cluster is 0.05. In Figure 4, the expected delay results are for $\alpha=0.3$, instead. Our results exhibit the significant delay advantage of the high priority traffic, as compared to that of the regular traffic, where this advantage increases as the traffic rate does. In addition, as the fraction of high priority traffic increases, the expected delays of the latter traffic decrease, while the effect of increasing number $M$ of monitored channels simultaneously decreases; in all cases, the latter effect is non-negligible only for relatively high traffic rates. For example, for $\alpha=0.3$, it suffices to assign a single neighboring channel to the high priority traffic $(M-1=1)$, for monitoring and possible transmission, where then the expected delays of the latter traffic never exceed 6.5 slots. 
Delay Slots

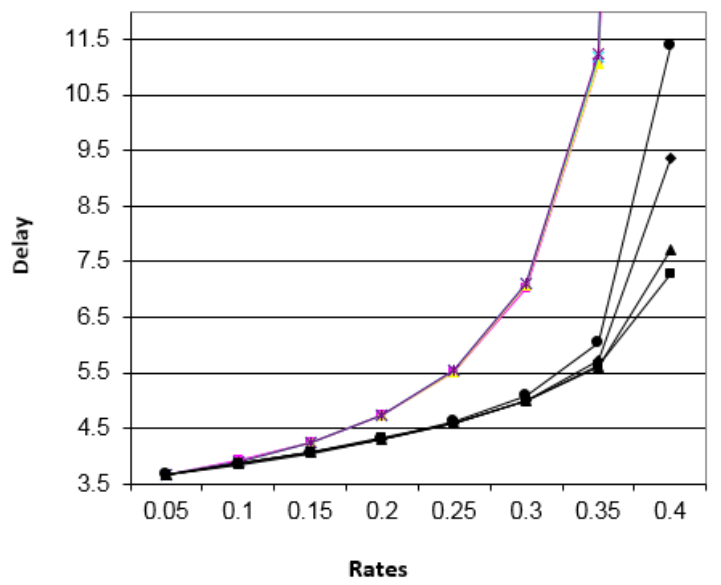

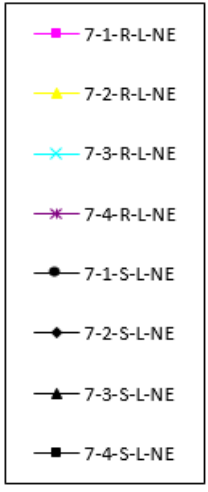

Figure 3: Expected delays in the absence of energy constraints as functions of cluster traffic rates, for $\alpha=0.05$

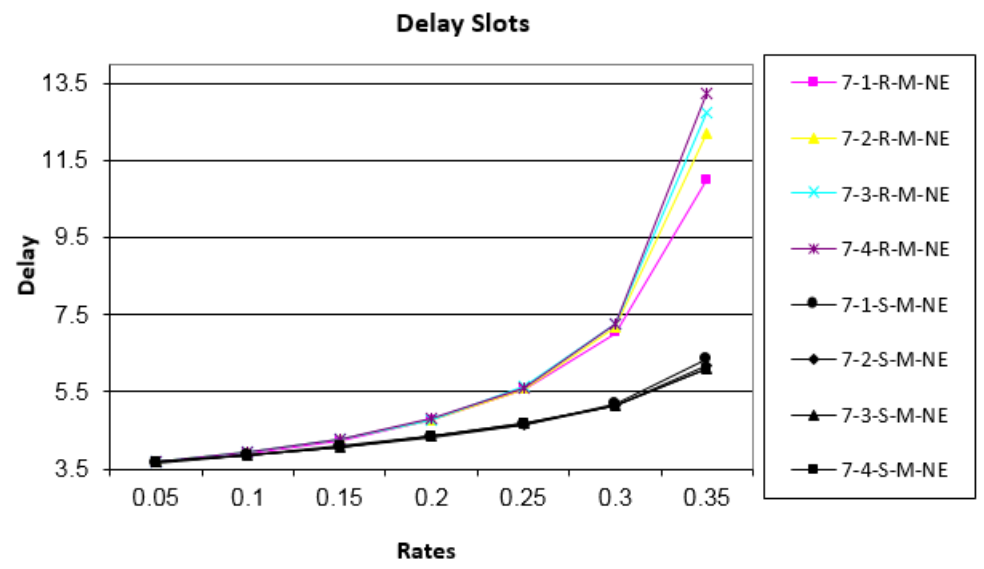

Figure 4: Expected delays in the absence of energy constraints as functions of cluster traffic rates, for $\alpha=0.3$

In the presence of sensor expirations, representative results are depicted by Figures 5 to 12 . The figures' legend is as that of Figures 3 and 4, where $E$ (instead of NE) stands here for the presence of energy constraints. Figures 5, 7, 9 and 11 display expected delays as functions of cluster traffic rates, while Figures 6, 8, 10 and 12 display rejection rates, instead. Figures 5, 6, 7 and 8 show results for $\alpha=$ 0.05 , while Figures $9,10,11$ and 12 exhibit results for $\alpha=0.3$. Figures 5, 6, 9 and 10 correspond to expiration constants' values $[\beta, \eta, \xi]=[5,10,100]$, while Figures $7,8,11$ and 12 correspond to $[\beta, \eta, \xi]$ $=[5,50,500]$, instead. From the results in the figures, we first observe that, in all cases, as the traffic cluster rate increases, expected delays stabilize when rejection rates are sufficiently large; for the case of $[\beta, \eta, \xi]=[5,50,500]$, where the stored per sensor energy is relatively high, the expected delays for the regular users take a dip at the point where the expected delays assume a sharp increase. As expected, in all cases, the rejection rates for the high priority users increase, as the number $M$ of monitored channels increases, since these users loose then increased energy in monitoring. As the stored per sensor energy increases (from $\xi=100$ to $\xi=500$ ), the expected delays increase; since less traffic is then rejected. At the same time, when the stored per sensor energy is relatively large, increase in the number $\mathrm{M}$ of monitored channels does not present the high priority users with a significant delay advantage, while it reduces significantly their rejection losses in the presence of relatively high cluster 
traffic rates and relatively low $\alpha$ values; when $\mathrm{M}$ is larger, the monitoring time by the high priority users is then sufficiently low to allow transmission before expiration. As compared to the regular users with relatively high energy reserves, high priority users possessing the same reserves are presented with a significant delay advantage and with simultaneously minimal losses; for cluster traffic rates below 0.4 , while they experience less significant delay and loses reductions; for cluster traffic rates above 0.4. The tradeoff between delays and rejection rates is strongly present when the stored per sensor energy is low, where, as the number $\mathrm{M}$ of monitored by the high priority users increases, delays for all users decrease and rejection rates increase, while the delay and rejection differences between regular and high priority users also increase; as $M$ increases, high priority traffic experiences increased delay advantages at the expense of also increases rejection losses.

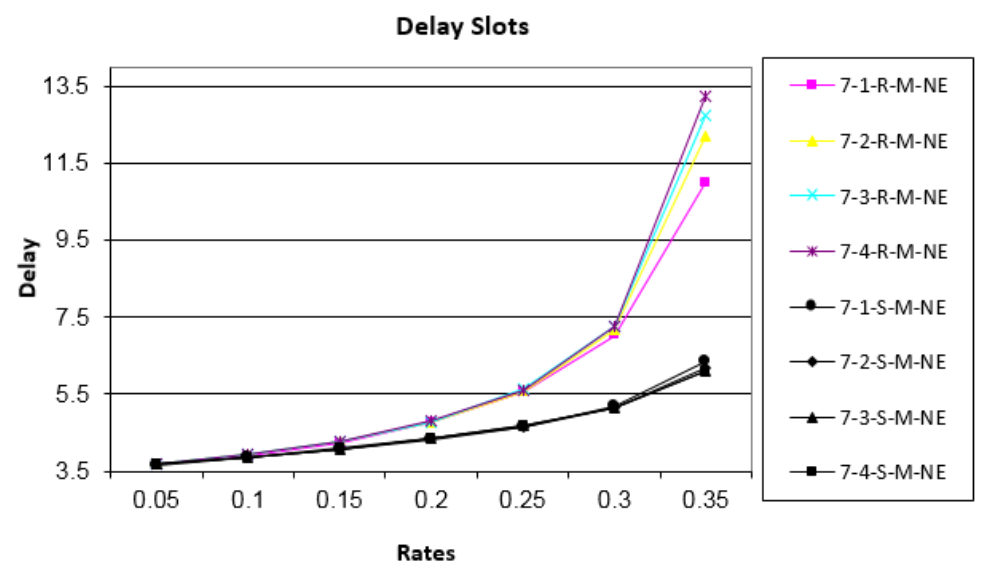

Figure 5: Expected delays in the presence of energy constraints as functions of cluster traffic rates, for $\alpha=0.05$ and $[\beta, \eta, \xi]=[5,10,100]$

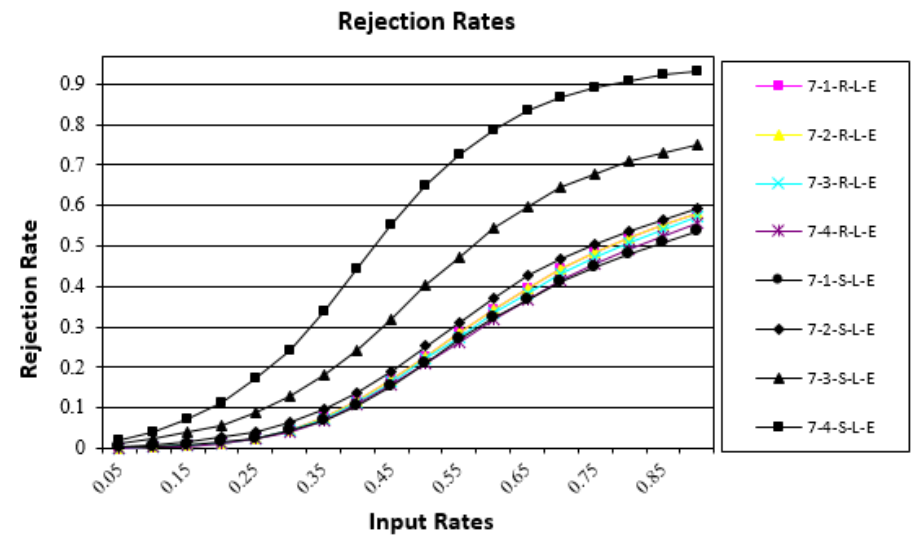

Figure 6: Rejection rates in the presence of energy constraints as functions of cluster traffic rates, for $\alpha=0.05$ and $[\beta, \eta, \xi]=[5,10,100]$ 


\section{Delay Slots}
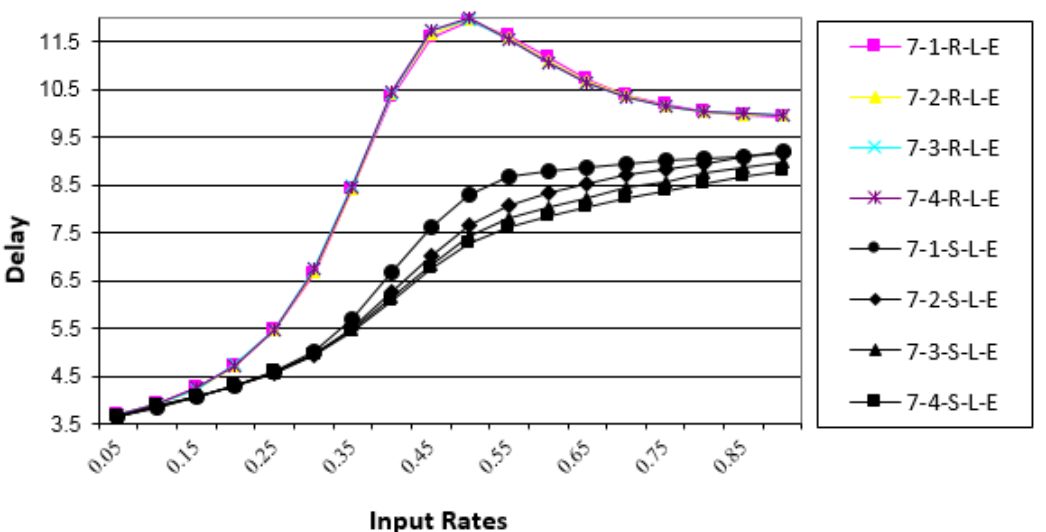

Input Rates

Figure 7: Expected delays in the presence of energy constraints as functions of cluster traffic rates, for $\alpha=0.05$ and $[\beta, \eta, \xi]=[5,50,500]$

\section{Rejection Rates}
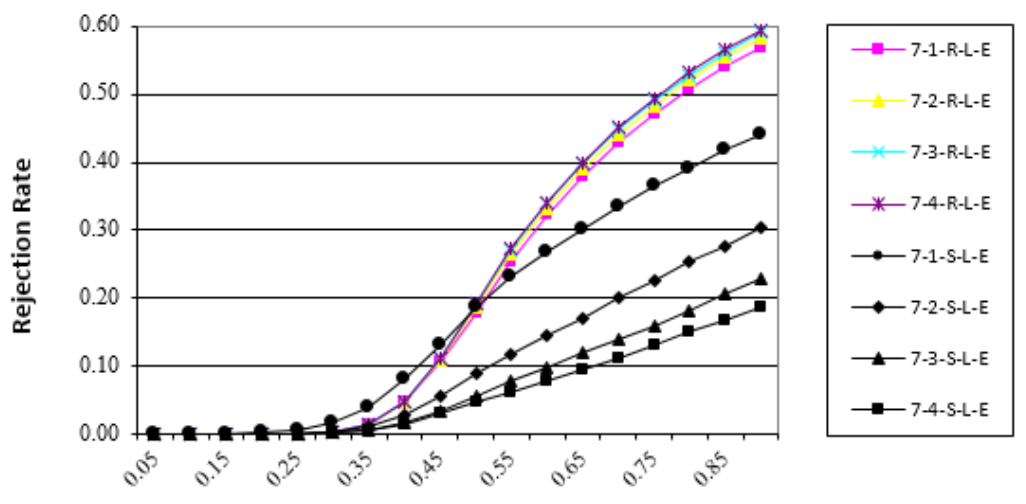

Input Rates

Figure 8: Rejection rates in the presence of energy constraints as functions of cluster traffic rates, for $\alpha=0.05$ and $[\beta, \eta, \xi]=[5,50,500]$

\section{Delay Slots}

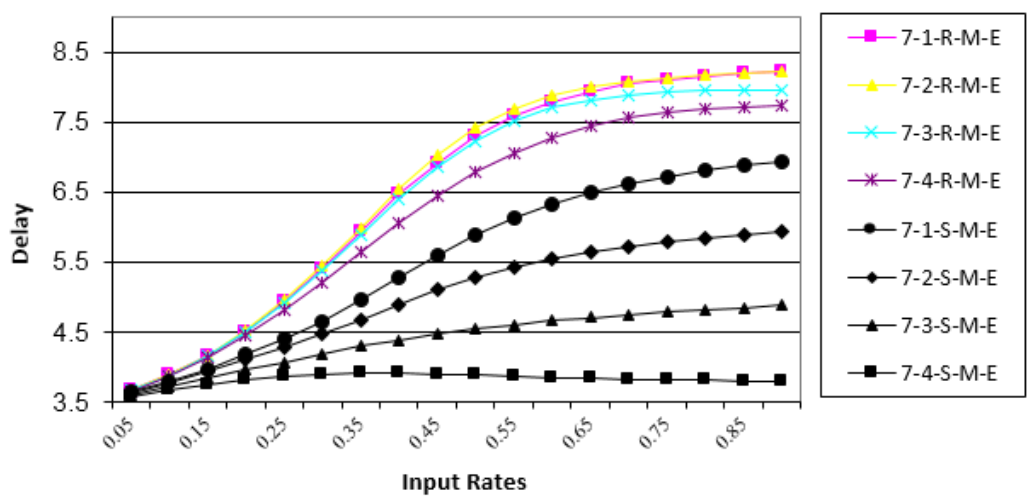

Figure 9: Expected delays in the presence of energy constraints as functions of cluster traffic rates, for $\alpha=0.3$ and $[\beta, \eta, \xi]=[5,10,100]$ 
Rejection Rates

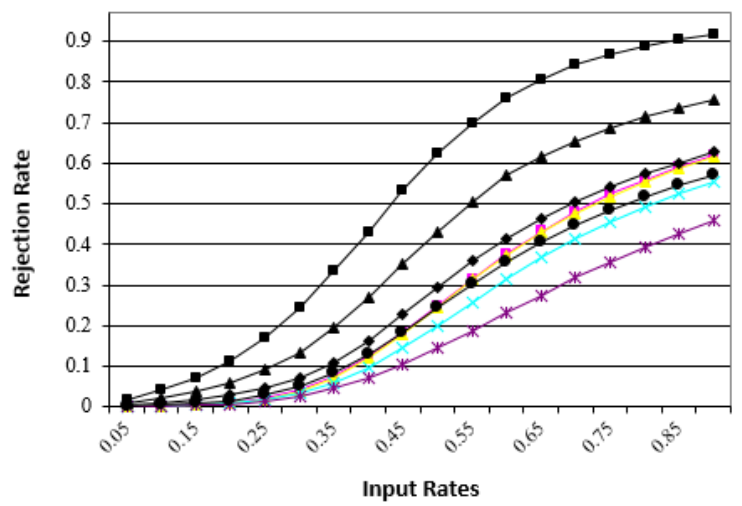

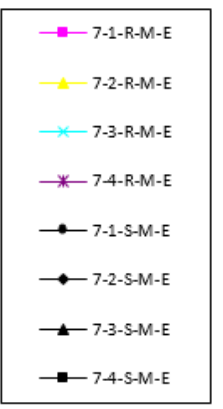

$-7-4-S-M-E$

Figure 10: Rejection rates in the presence of energy constraints as functions of cluster traffic rates, for $\alpha=0.3$ and $[\beta, \eta, \xi]=[5,10,100]$

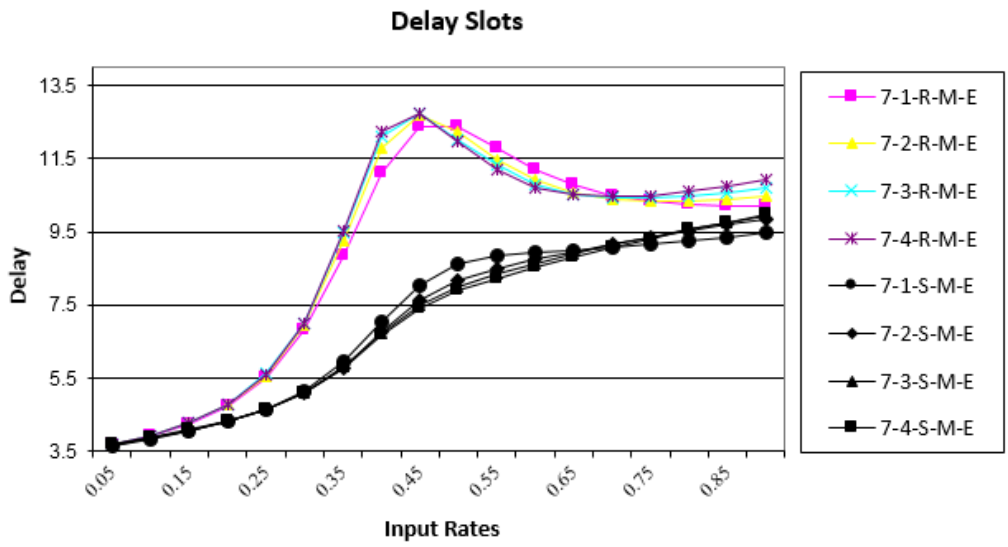

Figure 11: Expected delays in the presence of energy constraints as functions of cluster traffic rates, for $\alpha=0.3$ and $[\beta, \eta, \xi]=[5,50,500]$

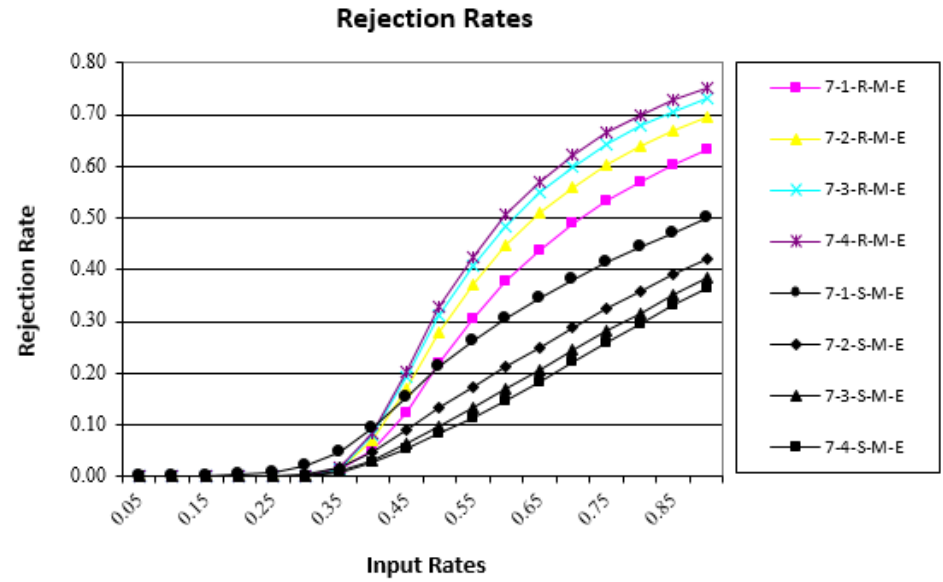

Figure 12: Rejection rates in the presence of energy constraints as functions of cluster traffic rates, for $\alpha=0.3$ and $[\beta, \eta, \xi]=[5,50,500]$ 


\section{Conclusions}

We analyzed and numerically evaluated a random access algorithm for deployment in clustered sensor networks which incorporate regular as well as high priority traffics, where high priority users monitor and occasionally transmit through neighboring cluster channels for delay reduction All sensors are assumed to contain limited energy reserves with energy consumed by channel monitoring and transmission attempts; such limited reserves inevitably induce traffic rejections. Our results show significant difference in system behavior between low level versus relatively high level of stored energy per sensor: (a) In the case of low level of energy reserves, a profound tradeoff between delays and traffic rejection rates presents itself. As the number of channels monitored by the high priority users increases, delays for all users decrease and rejection rates increase, where the delay and rejection rate differences between regular and high priority also increase: high priority users experience then lower delays, at the expense of increased rejection rates. (b) In the case of relatively high energy reserves, the system behavior may be distinguished between two regions of cluster traffic rates. For cluster traffic rates less than 0.4 , the delays of the high priority users are significantly lower than those of the regular users, while all users simultaneously experience low traffic rejection rates; increase in the number $\mathrm{M}$ of the monitored by the high priority users channels presents then no advantage, $M-1=1$ being recommended. For cluster traffic rates above 0.4 , the delays of the high priority users are not significantly lower than those of the regular users, while the rejection rates of the high priority users are then significantly lower than those of the regular users; the latter rejection rate reduction increases with increasing number $\mathrm{M}$ of monitored by the high priority users channels and decreasing fraction of high priority traffic.

\section{REFERENCES}

[1]. D. Blough and S. Paolo, "Investigating Upper Bounds on Network Life-Time Extension for Cell-Based Energy Conservation Techniques in Stationary Ad Hoc Networks", in Proc. ACM MobiCom, Atlanta, GA, Sep. 23-28, 2002, pp. 183-192.

[2]. M. Bhardwaj and A.P. Chandrakasan, "Bounding the Lifetime of Sensor Networks via Optimal Role Assignments", in Proc. IEEE INFOCOM, New York, Jun. 23-27, 2002, pp. 1587-1596.

[3]. J.H. Chang and L. Tassiulas, "Energy Conserving Routing in Wireless Ad Hoc Networks", in Proceedings of IEEE INFOCOM, Tel Aviv, Israel, Mar. 26-30, 2000, pp. 22-31.

[4]. Y. T. Hou, Y. Shi and H.D. Sherali, "On Node Lifetime Problem for Energy-Constrained Wireless Sensor Networks", ACM/Springer Mobile Netw.Applicat., vol. 10, no. 6, pp. 865-878, Dec. 2005.

[5]. Y.T. Hou, Y. Shi, J.H. Reed and K. Sohraby, "Flow Routing for Variable Bit Rate Source Nodes in EnergyConstrained Wireless Sensor Networks", in Proc. IEEE International Conference on Communications, Seoul Korea, May 16-20, 2005, pp. 3057-3062.

[6]. V. Rodoplu and T.H. Meng, "Minimum Energy Mobile Wireless Networks", IEEE J. Sel. Areas Commun., vol. 17, no. 8, Aug. 1999, pp. 1333-1344. 
A.T. Burrell \& P. Papantoni-Kazakos; Rate Dynamics and Transmission Algorithms in Clustered Sensor Networks, Transactions on Networks and Communications, Volume 2 No 5, Oct (2014); pp: 45-59

[7]. K. Sohrabi, J. Gao, V. Ailawadhi and G. Pottie, "Protocols for Self-Organizing of a Wireless Sensor Netrwork", IEEE Pers. Communications Magazine, vol. 7, Oct. 2000, pp. 16-27.

[8]. V. Srinivasan, P. Nuggehalli, C.F. Chiasserini and R. Rao, “Cooperation in Wireless Ad Hoc Networks", in Proc. IEEE INFOCOM, San Francisco, CA, Mar. 30-Apr. 3 2003, pp. 808-817.

[9]. R. Wattenhofer, L. Li, P. Bahl and Y.M. Wang, “Distributed Topology Control for Power Efficient Operation in Multihop Wireless Ad Hoc Networks", in Proc. IEEE INFOCOM, Snchorage, AK, Apr. 22-26, 2001, pp. 13881397.

[10]. A. T. Burrell and P. Papantoni Kazakos, "Performance Monitoring in Sensor Networks". ITNG 2009, April 2729, 2009, Las Vegas, Nevada.

[11]. Y. T. Hou, Y. Shi and H.D. Sherali, "Rate Allocation and Network Lifetime Problems for Wireless Sensor Networks", IEEE Trans. Networking, vol. 16, no. 2, April 2008, pp. 321-334.

[12]. D. Bertsekas and R. Gallager, Data Networks, Englewood Cliffs, NJ, Prentice Hall, 1992.

[13]. H. Luss and D.R. Smith, "Resource Allocation among Competing Activities: A Lexicigraphic Minimax Approach". Operations Research Letters, vol. 5, no. 5, Nov. 1986, pp. 227-231.

[14]. P. Papantoni Kazakos and A.T. Burrell "The Implementation of Dynamic Rate Allocation in Sensor Networks" Journal of Intelligent and Robotic Systems, 2010, Vol. 58, pp. 211-238.

[15]. A. T. Burrell and P. Papantoni-Kazakos, "Extended Sequential Algorithms for Detecting Changes in Acting Stochastic Processes", IEEE Trans. System, Man and Cybernetics, Vol. 28, no. 5, Sept. 1998, pp. 703-710.

[16]. A. T. Burrell, D. Makrakis, and P. Papantoni-Kazakos, "Traffic Monitoring for Capacity Allocation of Multimedia Traffic in ATM Broadband Networks", Telecommunication Systems, Vol. 9, July 1998, pp. 173206.

[17]. A.T. Burrell and P. Papantoni-Kazakos, "On-Line Learning and Dynamic Capacity Allocation in the Traffic Management of Integrated Services Networks", European Transactions on Telecommunications, Special Issue on Architectures, Protocols, and Quality of Service for the Internet of the Future, Vol. 10, No. 5, March/April 1999, pp. 202-214.

[18]. Fatma Salem, A.T. Burrell and P. Papantoni-Kazakos, "Dynamic Architectural Reconfigurations of Sensor Networks", IEEE IECON'2010, Glendale AZ, Nov. 7-10, 2010.

[19]. Fatma Salem, A.T. Burrell and P. Papantoni-Kazakos, "Dynamic Architecture- Reconfiguration Algorithms and Transmission Protocols for Clustered Sensor Network Topologies with Prioritized Data," International Scholarly Research Network (ISRN) Sensor Networks, 2012, Article ID 452981, 17 pages, doi: $10.5402 / 2012 / 452981$.

[20]. S. Bandyopadhyay and E.J. Coyle, "An Energy Efficient Hierarchical Clustering Algorithm for Wireless Sensor Networks", IEEE INFOCOM 2003.

[21]. D. Kazakos and P. Papantoni-Kazakos, Detection and Estimation, Computer Science Press, New York, NY, 1990. 
[22]. A. Papoulis and S.U. Pillai, Random Variables and Stochastic Processes, $4^{\text {th }}$ Edition, McGraw-Hill, New York, NY, 2002.

[23]. A.T.Burrell and T. Papantoni, "A Class of Limited Sensing Random Access Algorithms with Resistance to Feedback Errors and Effective Delay Control", Journal of Communications and Networks, 2006, Vol. 8, No. 1.

[24]. P. Papantoni-Kazakos, "Multiple Access Algorithms for a System with Mixed Traffic: High and Low Priority," IEEE Trans. Comm., March 1992, Vol. 40, pp. 541-555.

[25]. P. Papantoni-Kazakos, H. Delic, M. Paterakis, and M. Liu, "Transmission Algorithms for a Multi-Channel Packet Radio System with Priority Users," International Journal of Digital and Analog Communication Systems (IJDACS) John Wiley \& Sons, October-December 1993, Vol. 6, No. 4, pp. 193-212.

[26]. M. Paterakis and P. Papantoni-Kazakos, "A Simple Window Random Access Algorithm with Advantageous Properties," IEEE Trans. Inform. Th., September 1989, Vol. 35, pp. 1124-1130.

[27]. I. F. Akyildiz, W.Su, Y. Sankkarasubramaniam and E Cayirci, "Wireless Sensor Networks: A Survey", Computer Networks (Elsevier), vol. 38, no. 4, pp. 393-422, 2002.

[28]. A. T. Burrell and P. Papantoni-Kazakos, "Wireless Networks in Hybrid Topologies: Signaling, Transmission, and Dynamic Capacity Allocation Viewed in an Integrated Fashion," ITC Mini-Seminar Performance Modeling and Design of Wireless/PCS Networks, Cambridge, MA, October 3, 1996.

[29]. A.T. Burrell and P. Papantoni-Kazakos, "Random Access Algorithms in Packet Networks-A Review of Three Research Decades", International Journal of Communications Network and System Sciences (IJCNS), October 2012, Vol. 5, No. 10..

[30]. S. P. Meyn and R.L. Tweedie, Markov Chains and Stochastic Stability, Springer-Verlag, 1993.

[31]. M. Kaplan, "A Sufficient Condition for Nonergodicity of a Markov Chain”, IEEE Transactions on Information Theory, vol. 25, 1979, pp. 470-471. 